\title{
Editorial: The Biogeochemistry, Biophysics, Radiobiology, and Technical Challenges of Deep Subsurface Research
}

\author{
Geoffrey Battle Smith ${ }^{1 *}$, Maria Antonella Tabocchini ${ }^{2,3}$ and Carlos Peña Garay ${ }^{4}$ \\ ${ }^{1}$ Department of Biology, New Mexico State University, Las Cruces, NM, United States, ${ }^{2}$ Istituto Superiore di Sanità (ISS), \\ Rome, Italy, ${ }^{3}$ Istituto Nazionale di Fisica Nucleare (INFN) Sezione Roma 1, Rome, Italy, ${ }^{4}$ Laboratorio Subterráneo de \\ Canfranc, Canfranc, Spain
}

Keywords: subsurface science, radiation biology, biogeochemistry, below background radiation, biophysics, radiation health effects

Editorial on the Research Topic

The Biogeochemistry, Biophysics, Radiobiology, and Technical Challenges of Deep Subsurface Research

Numerous earth science projects are being carried out worldwide in the underground in both natural settings and laboratories. Life in the deep subsurface is dominated by microbes, which in turn directly impact the subsurface physicochemistry. The diversity of subsurface environments and interfaces that are able to sustain life, and the range of lifestyles that organisms have developed to exploit these systems give insight on how life has adapted to these unusual conditions, and

\section{OPEN ACCESS}

Edited and reviewed by:

Alexandra V. Turchyn

University of Cambridge,

United Kingdom

${ }^{*}$ Correspondence:

Geoffrey Battle Smith

gsmith@nmsu.edu

Specialty section: This article was submitted to Biogeoscience, a section of the journal

Frontiers in Earth Science

Received: 08 March 2021 Accepted: 09 April 2021

Published: 13 May 2021

Citation:

Smith GB, Tabocchini MA and Garay CP (2021) Editorial: The Biogeochemistry, Biophysics,

Radiobiology, and Technical Challenges of Deep Subsurface Research. Front. Earth Sci. 9:678034. doi: 10.3389/feart.2021.678034 this in turn illuminates potential conditions for life on other planets. As more labs, mines and other commercial facilities are installed internationally, the impacts of subsurface conditions on humans have become increasingly important. Model organisms from the surface ranging from bacterium to fly to fish and worm are brought underground to test the benefits or consequences to life underground. The absence of natural levels of radiation in the subsurface allows for the experimental testing of the hypothesis that background, surface levels of radiation maintain fitness. In this Research Topic, some articles document subsurface prokaryotic life extremes and others focus on the biological response and adaptation to deep subsurface environments as well as on the technical challenges associated with this research. This is a highly cross-disciplinary collection, reflecting the expertise required in Biology, Physics and Geochemistry to successfully work in this field.

The cover photos for this Research Topic show a researcher in the Italian cave, Grotta Grande dei Cervi, and a microbial consortium of iron oxidizers that colonize pyrite in Spain's Iberian Pyrite Belt (details reported in Vacarelli et al., and Escudero et al., respectively). The photos thus represent two of the many scales used in deep subsurface research, which utilizes measurements ranging from astronomical to subatomic. As an indication of the vitality of the discipline we note 14 deep underground laboratories worldwide (Ianni), with two international astroparticle physics labs (UK's Boulby and Canada's SNOLAB) recently starting up new underground Biology laboratories (Boulby Astrobiology Lab, "BISAL" reported by Wadsworth et al. and the REPAIR project at SNOLAB, reported by Pirkkanen et al.).

Papers on biogeochemistry, radiation biology, astrobiology, and methods in mitigating background radiation are covered in this collection. Escudero et al. report on the influence of pyrite on the distribution of the iron-oxidizing Acidovorax bacterial group using an innovative approach combining catalyzed reporter deposition fluorescence in-situ hybridization and fluorescence plus 
confocal Raman microscopy. Intriguing evidence that the Acidovorax species in these deep subsurface samples may oxidize pyrite mineral is presented. Vaccarelli et al. combine electron dispersive spectroscopy and scanning microscopy with DNA analysis to report on the association of cave manganese laminae and microbial biosignatures. These reports have application to identifying conditions that may support life in other planetary systems. A biogeochemistry paper with industrial application by Ren et al., reports on the microbial communities in oil production wells, demonstrating by pyrosequencing analysis that different methods of oil extraction significantly shift the oil field microflora.

A number of the radiation biology papers report on the remarkable biological response to below background radiation. Statistically significant responses of mammalian tissue culture (Liu et al.), whitefish (Pirkkanen et al.), fruit fly (Esposito et al.) and nematode (Van Voorhies et al.) provide evidence that multiple organismal models "notice" minute differences in radiation fields and respond to sub-background radiation levels at the proteomic, transcriptomic and physiological levels.

Some papers show how underground biology studies are relevant not only for improving our knowledge on life evolution, but also for assessing the robustness of the present radiation protection system that currently assumes that public health risk is linearly related to the radiation dose. The review by Belli and Indovina describes the mechanisms involved in the biological response to low dose and protracted exposures, particularly to natural background radiation and emphasizes how cell response to low doses appears more complex than that assumed for radiation protection purposes and is not always detrimental. Overall, data suggest the hypothesis that environmental radiation contributes to the development and maintenance of homeostatic balance and defense response in living organisms.

Further studies on pKZ1 A11 mouse hybridoma cells (Fischietti et al.) analyzed proliferation and expression of key proteins involved in stress response, apoptosis, and autophagy in underground and above ground environments. The results showed differential response to growth-stress conditions with a switch from apoptosis to autophagy, correlated with activation of $\mathrm{p} 53$, in the underground environment. They also suggest a scarce contribution of the gamma component of environmental radiation in triggering the biological response. This is an issue presently under investigation at the Laboratori Nazionali del Gran Sasso (LNGS, Esposito et al.). The RENOIR experiment, recently launched, is aimed at improving knowledge on the environmental radiation spectrum and to investigate the specific role of the gamma component on the biological response of Drosophila melanogaster.

Future low background biological experiments will require better control on the radioactive backgrounds. Underground laboratories provide a natural reduction of cosmic radiation, while radioactive decays from potassium-40 and the naturally occurring decay chains from uranium-238, uranium-235 and thorium-232 should be reduced by experimental design. Current most sensitive ultra-low background detection techniques and best level of background reduction are discussed by Laubenstein and Lawson, who coordinate a worldwide germanium detectors cross-calibration program. The gamma background is dominated by radon emanation, which is generally larger underground and should be reduced by ventilation. Further reduction requires the use of low radon cleanrooms. A detailed discussion of prototyping this installation is presented by Štekl et al., who discussed both the control of radon concentration in air and radon decay products on surfaces. Potassium-40 is singular among the radioactive isotopes because it is necessary for the function of all living cells. Tomasi discusses the interesting proposal for the therapeutic use of potassium enriched in the $\mathrm{K}-40$ isotope for patients suffering from diseases caused by mitochondrial dysfunctions.

\section{AUTHOR CONTRIBUTIONS}

All authors listed have made a substantial, direct and intellectual contribution to the work, and approved it for publication.

Conflict of Interest: The authors declare that the research was conducted in the absence of any commercial or financial relationships that could be construed as a potential conflict of interest.

Copyright (c) 2021 Smith, Tabocchini and Garay. This is an open-access article distributed under the terms of the Creative Commons Attribution License (CC BY). The use, distribution or reproduction in other forums is permitted, provided the original author(s) and the copyright owner(s) are credited and that the original publication in this journal is cited, in accordance with accepted academic practice. No use, distribution or reproduction is permitted which does not comply with these terms. 